\section{Identification of a homozygous recessive variant in PTGS1 resulting in a congenital aspirin-like defect in platelet function}

\author{
Melissa V. Chan,,$^{1^{*}}$ Melissa A. Hayman,,$^{1^{\star}}$ Suthesh Sivapalaratnam, ${ }^{2,3,4^{\star}}$ Marilena \\ Crescente, ${ }^{1}$ Harriet E. Allan, ${ }^{1}$ Matthew L. Edin, ${ }^{5}$ Darryl C. Zeldin, ${ }^{5}$ Ginger L. Milne, ${ }^{6}$ \\ Jonathan Stephens, ${ }^{2,3}$ Daniel Greene, ${ }^{2,3,7}$ Moghees Hanif, ${ }^{4}$ Valerie B. O'Donnell, ${ }^{8}$ \\ Liang Dong, ${ }^{9}$ Michael G. Malkowski, ${ }^{9}$ Claire Lentaigne,${ }^{10,11}$ Katherine \\ Wedderburn, ${ }^{2}$ Matthew Stubbs, ${ }^{10,11}$ Kate Downes, ${ }^{2,3,12}$ Willem H. Ouwehand, ${ }^{2,3,7,13}$ \\ Ernest Turro, ${ }^{2,3,7,12}$ Daniel P. Hart, ${ }^{1,4}$ Kathleen Freson, ${ }^{14}$ Michael A. Laffan, ${ }^{10,11 \#}$ \\ and Timothy D. Warner ${ }^{1 \#}$ \\ ${ }^{1}$ The Blizard Institute, Barts \& The London School of Medicine and Dentistry, Queen Mary \\ University of London, London, UK; ${ }^{2}$ Department of Hematology, University of Cambridge, \\ Cambridge, UK; ${ }^{3}$ National Health Service Blood and Transplant, Cambridge Biomedical \\ Campus, Cambridge, UK; ${ }^{4}$ Department of Hematology, Barts Health National Health \\ Service Trust, London, UK; ${ }^{5}$ National Institutes of Health, National Institute of \\ Environmental Health Sciences, Research Triangle Park, Durham, NC, USA; ${ }^{6}$ Division of \\ Clinical Pharmacology, Department of Medicine, Vanderbilt University Medical Center, \\ Nashville, TN, USA; ${ }^{7}$ Medical Research Council Biostatistics Unit, Cambridge Institute of \\ Public Health, Cambridge Biomedical Campus, Cambridge, UK; ${ }^{8}$ Systems Immunity \\ Research Institute, and Division of Infection and Immunity, School of Medicine, Cardiff \\ University, Cardiff, South Glamorgan, UK; ${ }^{9}$ Department of Structural Biology, Jacobs School \\ of Medicine and Biomedical Sciences, University of Buffalo, The State University of New \\ York, Buffalo, NY, USA; ${ }^{10}$ Imperial College Healthcare National Health Service Trust, \\ London, UK; ${ }^{11}$ Center for Hematology, Hammersmith Campus, Imperial College Academic \\ Health Sciences Center, Imperial College London, London, UK; ${ }^{12}$ NIHR BioResource, \\ Cambridge University Hospitals, Cambridge Biomedical Campus, Cambridge, UK; \\ ${ }^{13}$ Wellcome Trust Sanger Institute, Wellcome Trust Genome Campus, Hinxton, Cambridge, \\ UK and ${ }^{14}$ Department of Cardiovascular Sciences, Center for Molecular and Vascular \\ Biology, Gasthuisberg, University of Leuven, Leuven, Belgium \\ *MVC, MAH and SS contributed equally as co-first authors \\ "MAL and TDW contributed equally as co-senior authors
}

\section{ABSTRACT}

W Te have identified a rare missense variant on chromosome 9 , position 125145990 (GRCh37), in exon 8 in prostaglandin endoperoxide synthase 1 (PTGS1) (the gene encoding cyclo-oxygenase 1 [COX-1], the target of anti-thrombotic aspirin therapy). We report that in the homozygous state within a large consanguineous family this variant is associated with a bleeding phenotype and alterations in platelet reactivity and eicosanoid production. Western blotting and confocal imaging demonstrated that COX-1 was absent in the platelets of three family members homozygous for the PTGS1 variant but present in their leukocytes. Platelet reactivity, as assessed by aggregometry, lumi-aggregometry and flow cytometry, was impaired in homozygous family members, as were platelet adhesion and spreading. The productions of COXderived eicosanoids by stimulated platelets were greatly reduced but there were no changes in the levels of urinary metabolites of COX-derived eicosanoids. The proband exhibited additional defects in platelet aggregation and spreading which may explain why her bleeding phenotype was slightly more severe than those of other homozygous affected relatives. This is the first demonstration in humans of the specific loss of platelet COX-1 activity and provides insight into its consequences for platelet function and eicosanoid metabolism. Notably despite the absence of thromboxane $\mathrm{A}_{2}$ formation by platelets, urinary thromboxane $\mathrm{A}_{2}$ metabolites were in the normal range indicating these cannot be assumed as markers of in vivo platelet function. Results from this study are important benchmarks for the effects of aspirin upon platelet COX-1, platelet function and eicosanoid production as they define selective platelet COX-1 ablation within humans.
Haematologica 2021

Volume 106(5):1423-1432

\section{Correspondence:}

TIM WARNER

t.d.warner@qmul.ac.uk

Received: September 18, 2019.

Accepted: April 9, 2020.

Pre-published: April 16, 2020.

https://doi.org/10.3324/haematol.2019.235895

(C)2021 Ferrata Storti Foundation

Material published in Haematologica is covered by copyright. All rights are reserved to the Ferrata Storti Foundation. Use of published material is allowed under the following terms and conditions:

https://creativecommons.org/licenses/by-nc/4.0/legalcode. Copies of published material are allowed for personal or internal use. Sharing published material for non-commercial purposes is subject to the following conditions:

https://creativecommons. org//icenses/by-nc/4.0/legalcode, sect. 3. Reproducing and sharing published material for commercial purposes is not allowed without permission in writing from the publisher. 


\section{Introduction}

Platelets are central to the processes of hemostasis and thrombosis, the latter of which can lead to cardiovascular events such as myocardial infarction or stroke. At sites of vascular injury, platelets are activated upon interaction with collagen, von Willebrand factor (VWF) and fibrinogen and undergo shape change. In order to form a platelet plug, platelets first adhere, then pseudopodia extend from the surface. Following this, lamellipodia spread between these protrusions, resulting in a fully spread platelet within 30 minutes $(\mathrm{min}){ }^{1,2}$ Platelets release the contents of their dense and $\alpha$-granules, reinforcing activation and leading to the recruitment of further platelets to form a hemostatic plug in a positive feedback loop. ${ }^{3}$ A second feedback loop comprises liberation of arachidonic acid (AA) from membrane phospholipids by phospholipase $\mathrm{A}_{2}\left(\mathrm{PLA}_{2}\right)$ to form thromboxane $\mathrm{A}_{2}\left(\mathrm{TXA}_{2}\right)$.

AA is the substrate for three groups of eicosanoid-producing enzymes: lipoxygenase (LOX) which leads to hydroxyeicosatraenoic acids (HETE) and leukotrienes, cytochrome P450 (CYP450) which leads to epoxyeicosatrienoic acids (EET) and cyclo-oxygenase (COX) which leads to prostanoids. COX exists in two isoforms, the constitutively expressed COX-1 (more precisely known as prostaglandin endoperoxide synthase 1 [PTGS1]) and the (generally) inducible COX-2 [PTGS2], which both convert $\mathrm{AA}$ into prostaglandin (PG) G2 via an oxygenation reaction and then PGH2 via a peroxidase reaction. ${ }^{46}$ In platelets, PGH2 is then converted by thromboxane synthase to the pro-aggregatory $\mathrm{TXA}_{2}$. ${ }^{7} \mathrm{TXA}_{2}$ is a key part of the positive feedback loop mentioned above. Irreversible blockade of platelet COX-1 by aspirin abolishes the production of $\mathrm{TXA}_{2}$ by platelets, explaining its efficacy in antithrombotic prophylaxis. ${ }^{8}$ Because of aspirin's short half-life within the body and its irreversible effects upon COX, a low dose (75-100 mg per day) demonstrates a more selective effect upon platelets than upon the rest of the body, where nucleated cells can regenerate COX-1 protein. ${ }^{9-11}$

Here, we describe the first case of autosomal recessive inheritance of a rare variant in PTGS1 which reproduces the selective anti-platelet effect of aspirin and provides insight into the normal balance of prostanoid production.

\section{Methods}

Additional methods can be found in the Online Supplementary Appendix.

\section{Ethics and consent}

The proband and relatives were enrolled in the National Institute for Health Research (NIHR) BioResource under the Bleeding, Platelet and Thrombotic Disease domain after providing informed written consent ${ }^{12,13}$. The NIHR BioResource projects were approved by Research Ethics Committees in the UK and appropriate national ethics authorities in non-UK enrolment centers. Extensive phenotyping included coding of clinical and laboratory phenotypes with Human Phenotype Ontology (HPO) terms and collection of numerical and family history data was performed as described previously ${ }^{12}$. Healthy volunteer studies were approved by the NHS St. Thomas' Hospital Research Ethics Committee (07/O702/24). Healthy volunteers and family members abstained from non-steroidal anti-inflammatory drug (NSAID) use for 2 weeks before sample collection.

\section{Genotyping}

The proband and her parents underwent whole genome sequencing (WGS). Variants were called and annotated as described previously. ${ }^{13}$ In all other family members variants in PTGS1 were called by Sanger sequencing. ${ }^{14}$ Furthermore, the variant was expressed in cells and COX-activity was measured using a Clark type oxygen electrode. ${ }^{15}$

\section{Sample collection}

Midstream flow urine was collected and stored for subsequent eicosanomic analysis. ${ }^{16}$ Blood was collected by venepuncture into trisodium citrate (BD Diagnostics, UK). Platelet-rich plasma (PRP) was obtained by centrifugation at $175 x$ for 15 min. Platelet-poor plasma (PPP) was obtained by centrifugation of PRP at $12,000 \mathrm{xg}$ for $2 \mathrm{~min}$. COX-1 protein presence was determined by western blotting in platelets and confocal microscopy in platelets and leukocytes. In addition, the number of platelet-monocyte and platelet-neutrophil aggregates were quantified to assess whether this variant modulates interactions of platelets with other blood cells using an ImageStream ${ }^{\circledR} \mathrm{X}$ imaging flow cytometer (Merck Millipore, UK).

\section{Platelet function studies}

Platelet reactivity by light transmission aggregometry (LTA) and Optimul methods was completed within 2 hours of blood collection. ${ }^{17,18}$ In parallel, ATP release and P-selectin levels were determined to establish markers of platelets release and activation respectively and platelet spreading on collagen-coated surfaces were performed. ${ }^{19}$

\section{Data analysis}

Statistical summaries are presented as mean \pm standard deviation (SD). One-way ANOVA was performed using GraphPad Prism version 8.1.1 for Mac OS X (GraphPad Software, CA, USA) where appropriate. Statistically significant differences in means are presented as ${ }^{*} P<0.05,{ }^{* *} P<0.01,{ }^{* * *} P<0.001$ or ${ }^{* * * *} P<0.0001$. Percentiles of control values were generated, and pedigree member data was compared to this where only one value was obtained.

\section{Results}

\section{Whole genome sequencing and phenotyping of the pedigree}

The proband, a female of Iranian descent, aged 37 at enrolment (Figure 1, IV-1) was referred to the hemophilia outpatient clinic because of perioperative bleeding following a sinus operation. She had a history of cystic fibrosis (CF), C6 complement deficiency resulting in chronic infections, $\beta$-thalassemia trait and normoprolactinemic galactorrhoea. She had more extensive hemoptysis than expected from her CF and also suffered from frequent nosebleeds. At presentation she had a normal platelet count of $234 \times 10^{9} / \mathrm{L}$.

Upon taking the family history we appreciated that she was part of a large consanguineous family. Moreover, her mother (III-2) and maternal aunt (III-1) also had a clinical bleeding phenotype including easy bruising and menorrhagia. The two uncles (III-4 and III-5) and a cousin (IV-2) did not have any clinical bleeding (Figure $1 \mathrm{~A}$ and $\mathrm{B}$ ). Depending on the severity of bleeding, the proband received desmopressin, tranexamic acid and, very occasionally, platelets.

Sequencing demonstrated that the proband (IV-1), her mother (III-2) and aunt (III-1) were homozygous for a vari- 

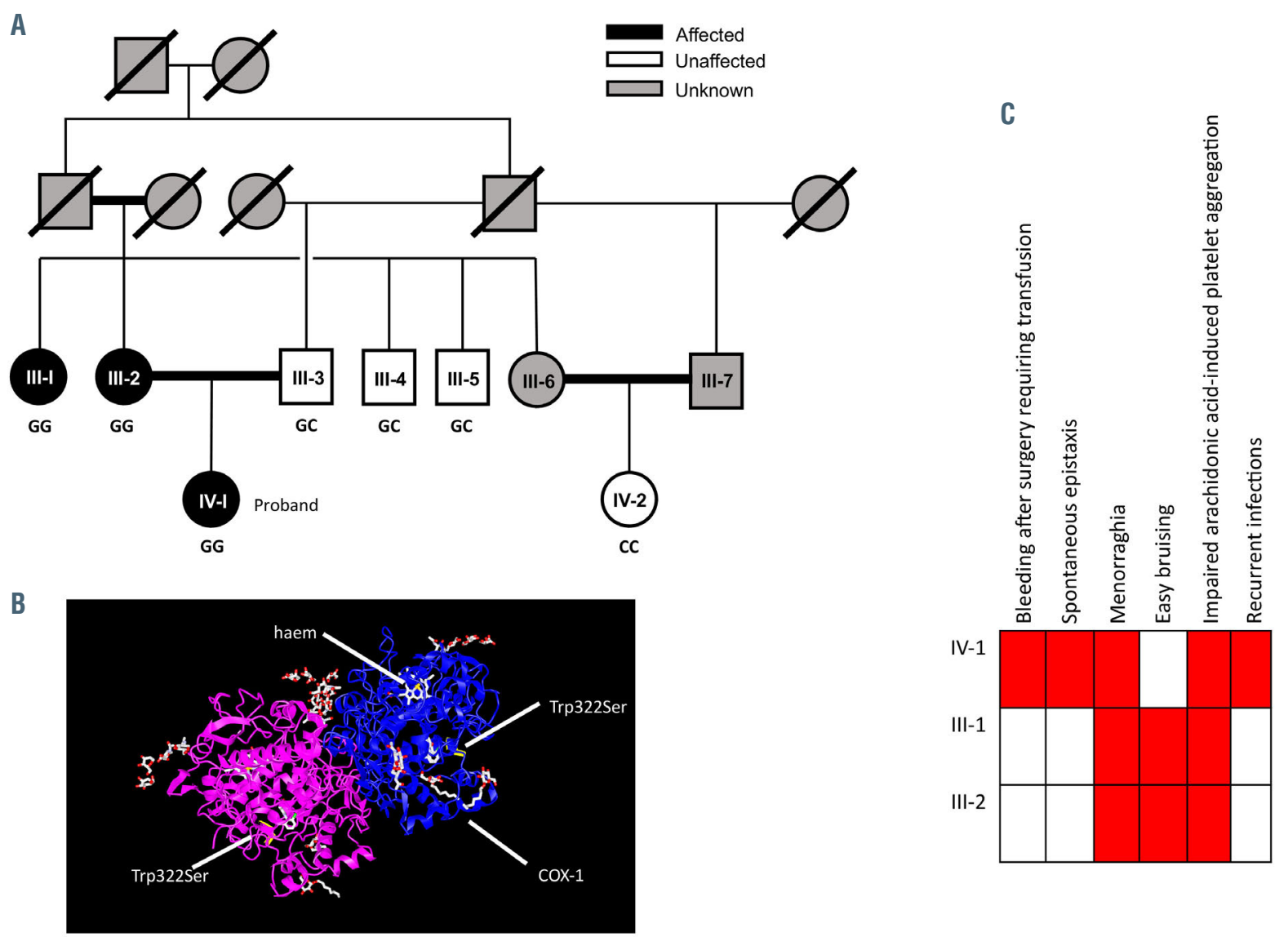

Figure 1. Pedigree phenotype. (A) Pedigree of the affected family, in which black, white and grey symbols indicate presence of the bleeding phenotype, absence of the bleeding phenotype and unknown bleeding phenotype, respectively. The genotype, where known, is shown under each symbol, where $\mathrm{G}$ is the mutant allele and $\mathrm{C}$ is the reference allele. Double lines indicate consanguinity and strike-through lines are used to indicate deceased individuals. (B) Human Phenotype Ontology (HPO) annotation of the three affected family members. (C) A ribbon diagram of the crystal structure of aspirin-acetylated cyclo-oxygenase 1 (COX-1) showing the location of the variant for the proband which results in a missense substitution of tryptophan to serine at amino acid 322.

ant on chromosome 9, position 125145990 (GRCh37), altering a guanine to a cysteine in exon 8 of PTGS1. This variant resulted in a missense substitution of tryptophan to serine at amino acid 322 (Figure 1C). The variant had a Combined Annotation Dependent Depletion (CADD) score $^{20}$ of 31.0 and was absent from the Genome Aggregation Database (gnomAD). ${ }^{21}$ Using Alamut ${ }^{\circledR}$ Visual, the new variant has been shown to be highly conserved with a phyloP score of 9.88. III-3, III-4 and III-5 were heterozygous (GC) for the alternate allele while IV-2 was homozygous for the reference allele (CC), where $\mathrm{C}$ represents the wild-type and $\mathrm{G}$ represents the mutant allele. III6 and III-7 were unavailable for genotyping. The proband had an additional biallelic mutation (chromosome 7 , position 117175467) causing a splice donor variant in the CF transmembrane conductance regulator (CFTR) gene causing CF.

\section{COX-1 protein in platelets and leukocytes}

COX-1 protein in platelet lysates from the proband and her homozygous relatives was absent. In III-3 and III-4, expression was present but reduced and was at normal levels in III-5 and IV-2 (Figure 2A). The absence of COX-1 protein in platelets from the proband and homozygous relatives (Figure 2Ci and $\mathrm{Di}$ ) compared to a healthy control (Figures 2Bi) was confirmed with immunohistochemical analysis. COX-1 expression, however, was retained in leukocytes from all those tested (Figure 2Cii and Dii). The variant did not affect COX enzyme activity as shown in kinetic analysis of isolated recombinant protein (wildtype, $\mathrm{K}_{\mathrm{m}}=7.9 \pm 0.8 \mu \mathrm{mol} / \mathrm{L} ; \mathrm{W} 322 \mathrm{~S}, \mathrm{~K}_{\mathrm{m}}=14.1 \pm 1.1 \mu \mathrm{mol} / \mathrm{L}$, Online Supplementary Figure $S 1 A$ and $\mathrm{B}$ ). The variant also had no effect on COX activity after inhibition by aspirin (Online Supplementary Figure S1C). Though there was no appreciable phenotypic difference in the quality of interactions observed, there was a reduction in the number of platelet interactions with monocytes in the proband that was not found in other family members (controls, $34.8 \pm 19.2 \%$; proband, $7.5 \%$; homozygous relatives, $27.4 \pm 9.0 \%$; unaffected relatives, $32.8 \pm 10.6 \%$; Figure $3 \mathrm{~A}$ and $\mathrm{C}$ ). There was no change in platelet-neutrophil interactions (Figure $3 \mathrm{~B}$ and $\mathrm{D}$ ).

\section{The role of PTGS1 recessive variant on platelet reactivity}

Platelet reactivity is measured in vitro by aggregation and release experiments. Aggregation responses to arachidonic $\operatorname{acid}(\mathrm{AA} ; 1 \mathrm{mmol} / \mathrm{L}$ ) in the proband and her homozygous relatives were reduced compared to control from $65 \pm 7 \%$ to $4 \pm 1 \%$; responses to collagen $(1 \mu \mathrm{g} / \mathrm{mL}$ and $3 \mu \mathrm{g} / \mathrm{mL})$ reduced from $64 \pm 13 \%$ to $17 \pm 10 \%$ and from $67 \pm 8 \%$ to $20 \pm 11 \%$, respectively; and responses to adenosine diphosphate $(\mathrm{ADP})$ at $10 \mu \mathrm{mol} / \mathrm{L}$ was reduced from $62 \pm 11 \%$ to $38 \pm 13 \%$. Interestingly, the proband also had a greatly 
reduced response to ristocetin $(1.5 \mathrm{mg} / \mathrm{mL}$; control, $69 \pm 10 \%$; proband, $7 \%$ ) which was normal in all family members tested. Other than a reduced epinephrine $(10$ $\mu \mathrm{mol} / \mathrm{L})$ response, which was found in all family members, there was no difference in other LTA responses of the unaffected family members compared to control (Figure 4A). These findings were also reflected using Optimul aggregometry where AA responses were absent, and collagen and epinephrine responses were severely blunted in the proband and homozygous relatives (Online Supplementary Figure 2). TRAP-6 amide (25 $\mu \mathrm{mol} / \mathrm{L})$-stimulated ATP release was normal in all family members. AA $(1 \mathrm{mmol} / \mathrm{L})$ and collagen $(3 \mu \mathrm{g} / \mathrm{mL})$-stimulated secretion, however, was below the 20 th percentile in the proband and homozygous relatives (Figure 4B). Upon activation, platelets express $\mathrm{P}$-selectin and undergo shape change and spreading. U46619 (0.5 $\mu \mathrm{mol} / \mathrm{L})$-induced P-selectin expression was similar in all individuals (Figure 4C).

\section{The effect of the PTGS1 variant on platelet spreading}

The number of platelets with filopodia were increased in the proband and homozygous family members (control, $9 \pm 6 \%$; proband, $36 \pm 17 \%, P<0.001$; homozygous relative, $36 \pm 4 \%, P<0.01)$. The PTGS1 variant was also associated with a reduction in fully spread platelets (control, $45 \pm 6 \%$; proband, $9 \pm 9 \%, P<0.001$; homozygous relatives, $22 \pm 10 \%, P<0.001)$. Adherent platelets and number of lamellipodia were similar across all individuals tested (Figure 5). In addition, there were fewer platelets from both the proband and the homozygous relative that adhered to the fibrinogen-coated coverslips (control, $26 \pm 4 \%$; proband, $8 \pm 2 \%, P<0.001$; homozygous relatives, $13 \pm 3 \%, P<0.01)$.
The role of PTGS1 variant on eicosanoid production by stimulated whole blood and basal urine metabolites

Incubation of blood from healthy volunteers with collagen or TRAP- 6 amide greatly increased the levels of $\mathrm{TXB}_{2}$ (a stable breakdown product of $\mathrm{TXA}_{2}$ ), 11-dehydro-TXB (11-dH-TXB, a dehydrogenation product of $\left.\mathrm{TXB}_{2}\right)^{22}$, $\mathrm{PGE}_{2}, \mathrm{PGD}_{2}, 15$-HETE, 11-HETE and 12-HETE. In the PTGS1-deficient proband 12-HETE production was unaffected but there was an absence of $\mathrm{TXB}_{2}, \mathrm{PGE}_{2}, \mathrm{PGD}_{2}$ and 15-HETE (Figure 6A and B; Online Supplementary Table S1).

Despite the fact that platelets are able to synthesize $\mathrm{PGD}_{2}, \mathrm{PGE}_{2}$ and $\mathrm{TXA}_{2}$ from $\mathrm{PGH}_{2}$, urinary metabolites for these enzymes were unchanged in the proband and homozygous relatives compared to normal reference ranges. As expected, $\mathrm{PGI}_{2}$ metabolites, generated by $\mathrm{PGI}_{2}$ prostacyclin synthase from $\mathrm{PGH}_{2}$ in endothelial cells only were all within the standard range (Figure 6C to F; Online Supplementary Table S1). Indeed, leukocytes and endothelial cells are additional sources of $\mathrm{PGD}_{2}$ and $\mathrm{PGE}_{2}$ products, respectively.

\section{Discussion}

We report autosomal recessive inheritance of a homozygous rare missense variant in PTGS1 associated with an aspirin-like platelet phenotype. This phenotype provides the opportunity to definitively assess the roles of platelet COX-1 in human platelet function, including the production of eicosanoids. This cannot be assumed from exposure of platelets from other humans to aspirin in vivo or in vitro as aspirin has effects at sites other than platelet COX $-1^{23}$
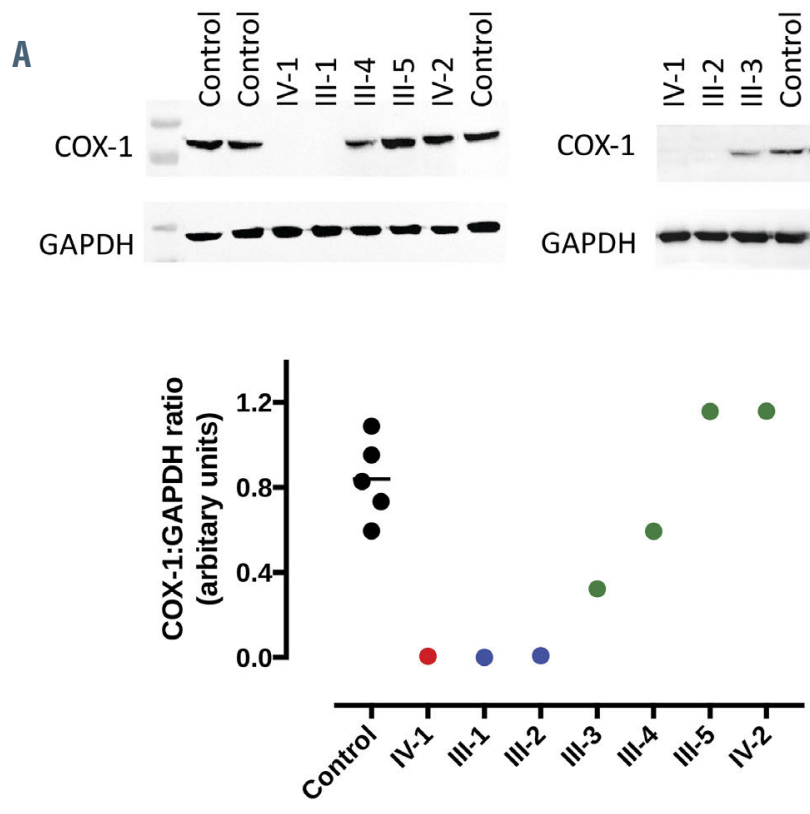

- Controls

- Homozygous relatives

- Proband
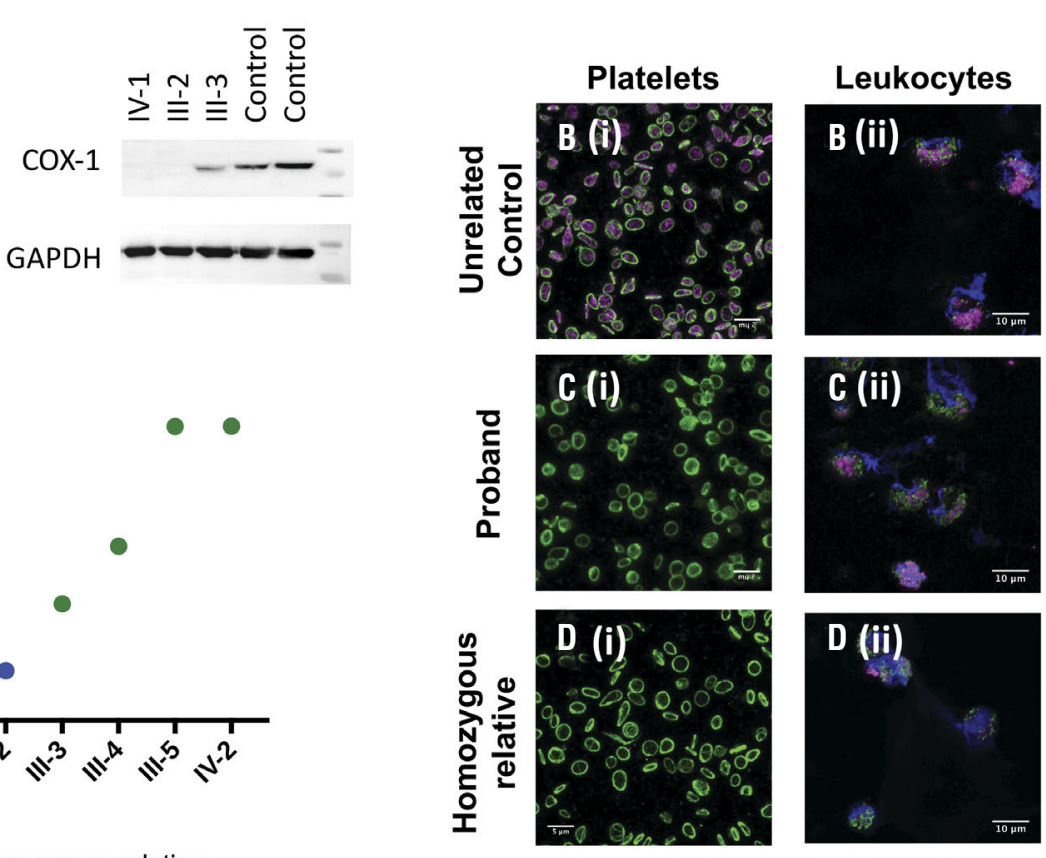

COX-1 Tubulin

COX-1 DAPI LAMP-3

Figure 2. coX-1 protein in control, proband and relatives. (A) Western blots and quantification of cyclo-oxygenase 1 (COX-1) and glyceraldehyde 3-phosphate dehydrogenase (GAPDH) expression in platelet lysates, isolated from the controls, the proband (IV-1), homozygous (III-1 and III-2) and unaffected (III-3, III-4, III-5 and IV2) family members. Representative immunohistochemical analysis of COX-1 expression in (B) control, (C) proband and (D) homozygous relative (i) platelets and (ii) leukocytes. Washed platelets were identified by tubulin (green) staining and COX-1 (magenta) was present in control but not in the proband or affected relative. In washed leukocytes, nuclear staining was confirmed by DAPI (blue), LAMP-3 (green) and COX-1 (magenta) was expressed in all samples. 
The $965 \mathrm{G}>\mathrm{C}$ variant of PTGS1 found in the family reported here is absent from gnomAD. In the identified pedigree, however, three of the eight family members studied were homozygous due to consanguinity and three were heterozygous for the variant. Interestingly, since this is a missense variant outside both the functional sites of the COX enzyme the phenotype was unexpected. Within the homozygous carriers, despite similar reproducible platelet aggregation, we saw minor differences in the bleeding phenotype, which reflects the clinical heterogeneity of presentation of some of the rare platelet disorders. This is also consistent with observations that while millions of people take aspirin daily to prevent secondary cardiovascular events, and this increases their risk of bleeding, the vast majority do not suffer from major spontaneous bleeding. Similarly, mice with a deficiency in COX-1 exhibit impaired haemostasis but only after being challenged by the tail-bleeding assay. ${ }^{24,25}$

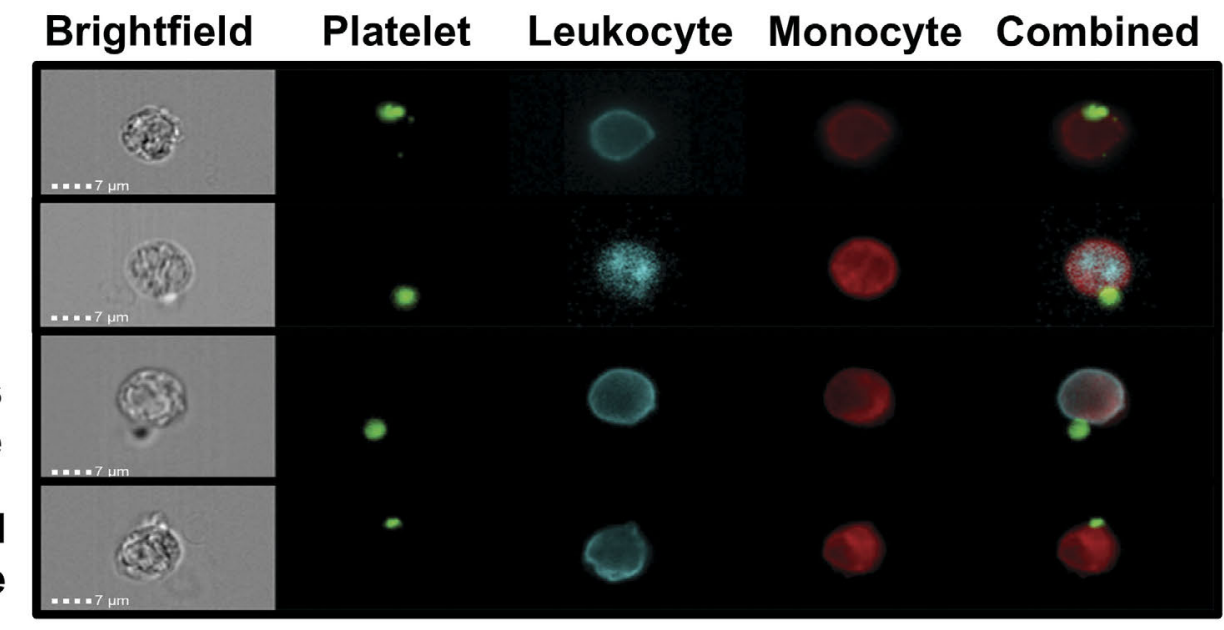

B

\section{Homozygous relative \\ Unaffected relative}

\section{Unrelated \\ Homozygous relative \\ Unaffected relative}

Brightfield

Platelet

Leukocy
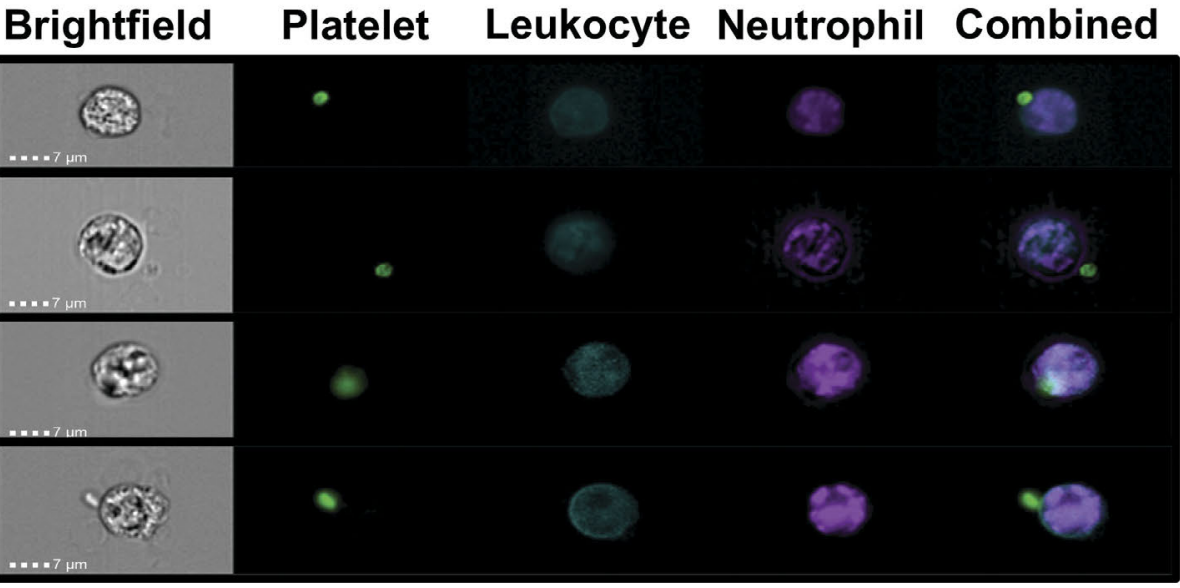

C

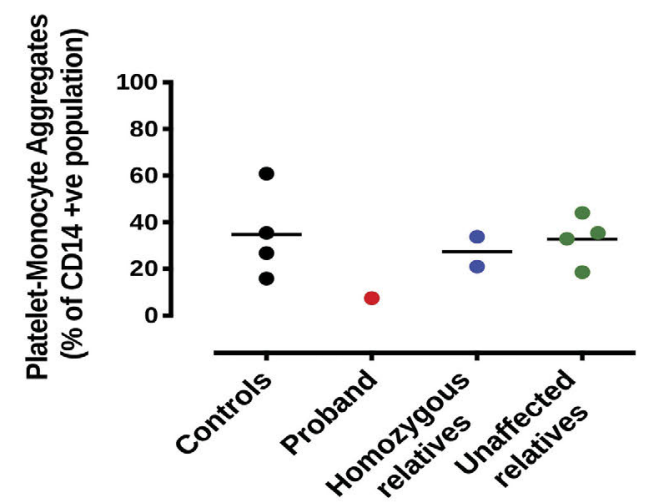

D

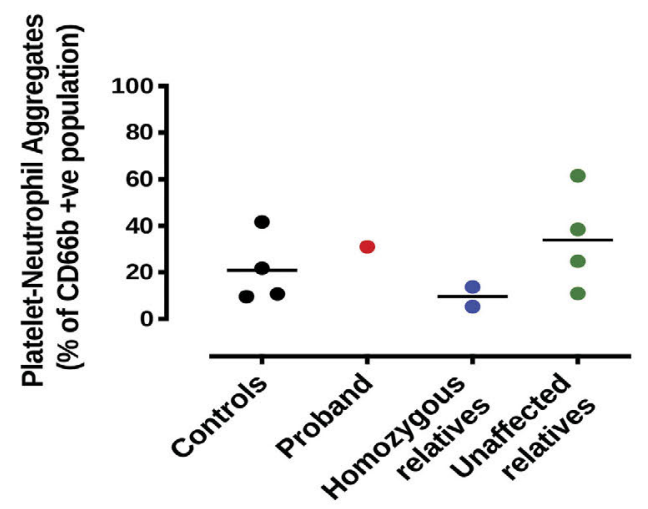


As above, data derived from the three pedigree members with the homozygous variant demonstrated a consistent effect upon platelet function and eicosanoid profile, irrespective of other clinical differences. Notably, the proband had an additional diagnosis of CF and administration of COX inhibitors, which have anti-inflammatory effects, has been shown to inhibit the decline of lung function. ${ }^{26}$ However, the proband did not show any evidence of a beneficial effect accruing from the absence of her platelet COX-1, in keeping with our understanding of the anti-inflammatory effects of NSAID being mediated primarily via inhibition of COX $-2 .^{27}$

While COX-1 protein was expressed at normal levels in platelets from the family member with the CC genotype, it was absent in those with the GG genotype. Conversely, COX-1 was still expressed in the leukocytes irrespective of genotype. This may indicate that the PTGS1 variant is not expressed by megakaryocytes or that the variant affects the stability of the protein; i.e., that the COX-1 protein degrades more rapidly and then cannot be replenished within platelets because they lack transcriptional machinery, akin to what is observed in erythrocytes in glucose-6-phosphate dehydrogenase deficiency ${ }^{28}$. Inheritance of one copy of the mutant allele resulted in variable but never absent platelet COX-1 protein levels which were sufficient to sustain function. When the variant was expressed and characterized, the recombinant protein was found to have normal enzyme activity which is consistent with the findings that in homozygous family members urinary COX-1 metabolites where within the normal range; i.e., implying that despite the PTGS1 variant, COX-1 activity in tissues other than the platelet was preserved. Due to constraints in sample availability, we were unable to investigate COX-1 protein levels in other nucleated cell types in the homozygous family members.

Platelet reactivity in the homozygous family members was consistent with that seen in previous studies in the presence of aspirin in vitro and in vivo. ${ }^{18,29,30}$ In particular, aggregation responses to collagen, epinephrine and ADP using both light transmission and Optimul aggregometry were reduced and responses to AA were absent but were normal to U46619 (TXA 2 analogue). Homozygous knockout mice for PTGS1 show similar impairment in platelet aggregation. $^{31,32}$ ATP release from dense granules induced by collagen was impaired in platelets from homozygous family members which is similarly concordant with an aspirin-like defect ${ }^{29}$.

Platelet spreading in all homozygous family members was impaired. Specifically, the number of actin-rich filopodia was increased, though the number of platelets which reached the point of being fully spread was lower. Indeed, the number of platelets which adhered to the fibrinogen-coated surface was significantly reduced, indicating a dysfunction in the process leading to formation of a stable platelet plug which could increase the risk of bleeding. This evidence suggests that either this variant or an unknown defect carried by these family members is associated with a dysfunction in the signaling mechanisms required for sufficient spreading. Whilst we did not directly compare platelet spreading from the homozygous family with that of low-dose aspirin-treated healthy subjects,

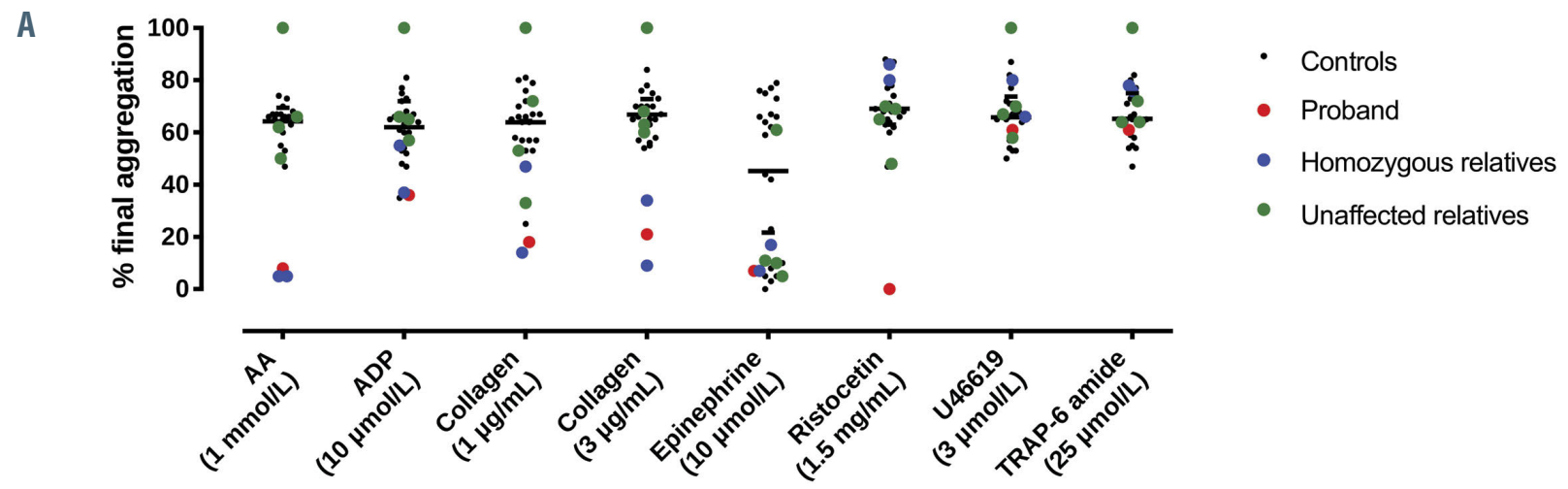

B

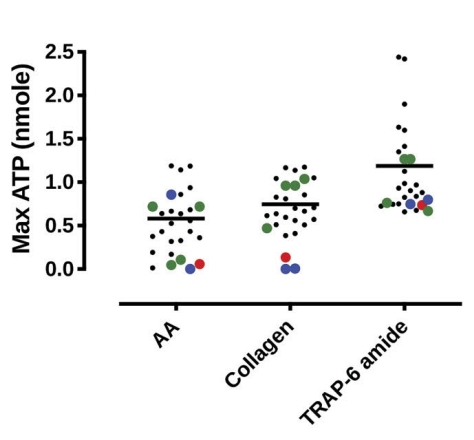

C

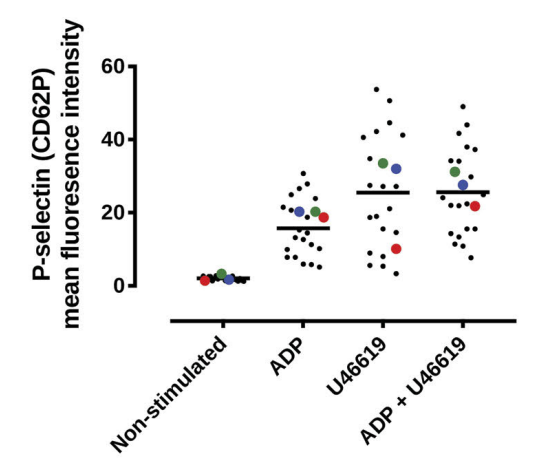

Figure 4. Effect of PTGS1 variant on platelet aggregation, secretion, and adhesion responses. (A) Aggregation responses to arachidonic acid (AA; 1 mmol/L), adeno-

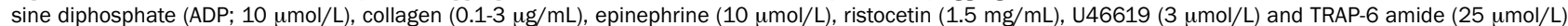
and (B) ATP secretion to AA $(1 \mathrm{mmol} / \mathrm{L})$, ADP $(10 \mu \mathrm{mol} / \mathrm{L})$, collagen $(3 \mu \mathrm{g} / \mathrm{mL})$ and TRAP- 6 amide $(25 \mu \mathrm{mol} / \mathrm{L})$. $n=20$ (healthy controls; range with median); $n=1$ (proband); $n=2$ (homozygous relatives); $n=4$ (unaffected relatives). (C) P-selectin expression as measured by flow cytometry in whole blood stimulated by ADP (40 $\mu \mathrm{mol} / \mathrm{L})$, U46619 (0.5 $\mu \mathrm{mol} / \mathrm{L})$ or ADP plus U46619. 

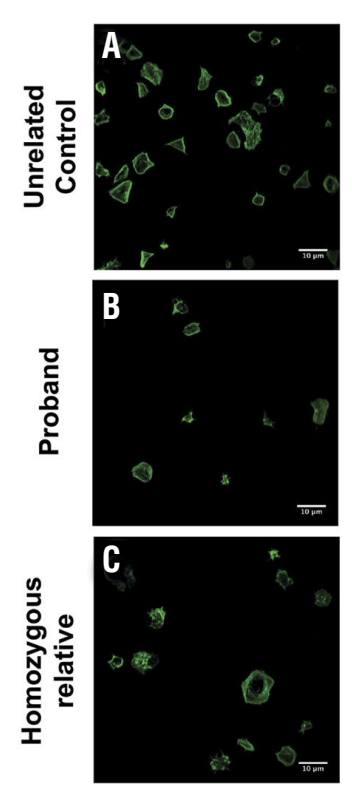

D

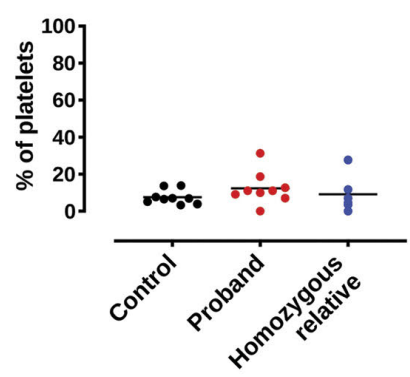

Lamellipodia

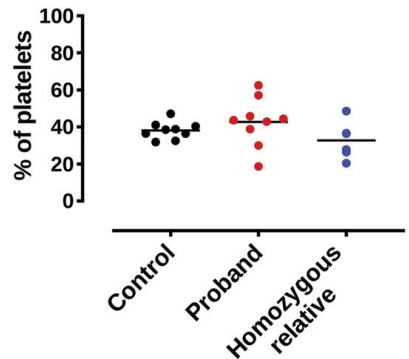

Filopodia

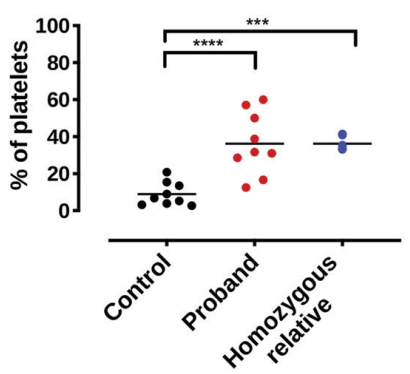

Fully Spread

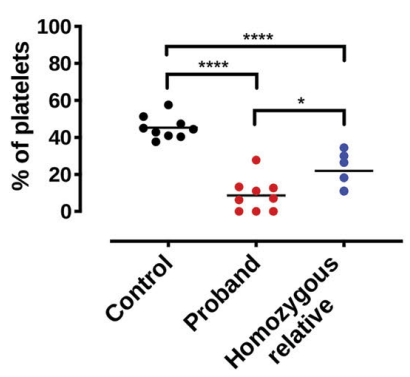

Total
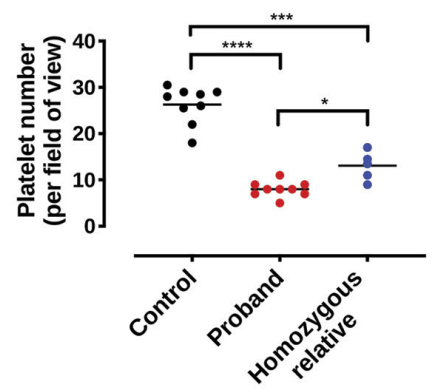

Figure 5. Platelet spreading on fibrinogen-coated surfaces. (A) a control, (B) the proband and (C) a homozygous relative with (D) quantification of adhered platelet, filopodia, lamellipodia, fully spread platelet frequency and total platelets per field of view.

A
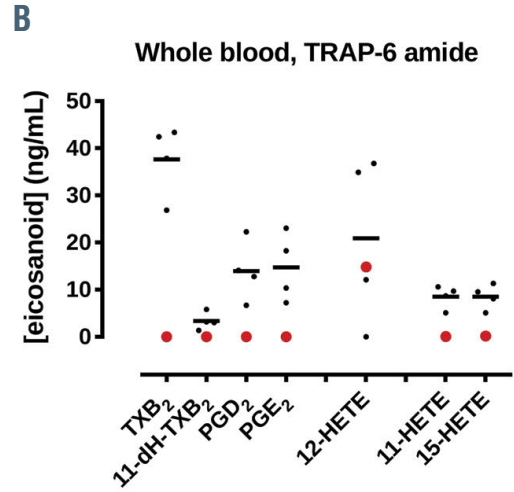

C

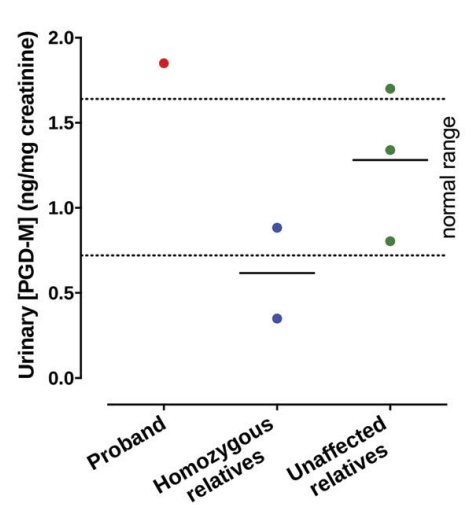

D

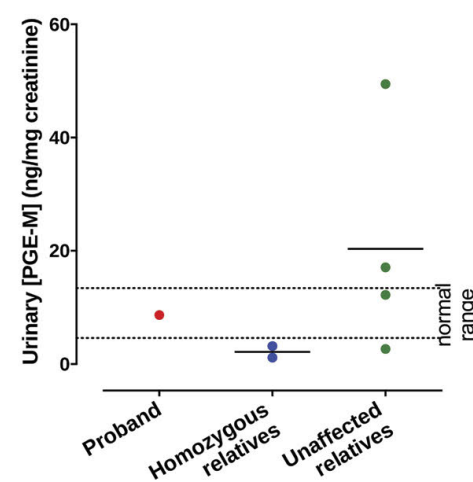

$\mathrm{E}$

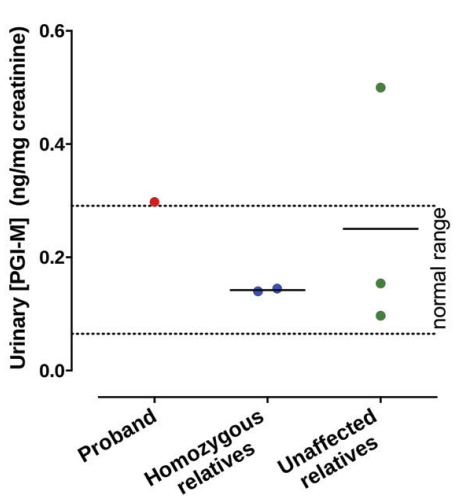

$F$

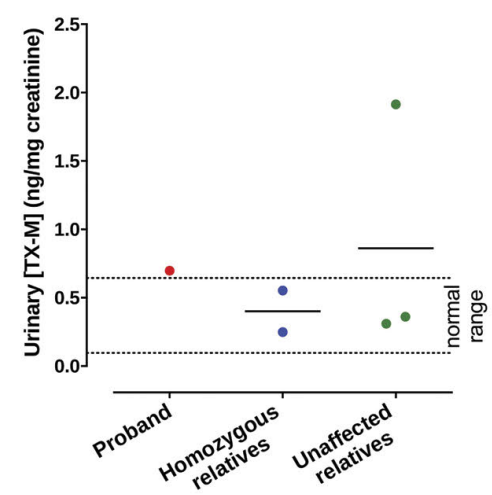

- Controls

- Proband

Figure 6. Contribution of PTGS1 to eicosanoid synthesis in whole blood and urine. Platelet-derived eicosanoid levels in whole blood from healthy volunteers or from the proband stimulated with collagen $(30 \mathrm{mg} / \mathrm{mL})(A)$ or TRAP- 6 amide $(30 \mu \mathrm{mol} / \mathrm{L})(\mathrm{B})$. Levels are expressed as increase over levels in vehicle-treated blood. Urinary (C) prostaglandin (PG) $D_{2}$ and (D) $P G E_{2}(C) P G I_{2}$ and (D) thromboxane $A_{2}\left(T_{X} A_{2}\right.$ ) metabolite levels in proband, homozygous and unaffected relatives. $n=4$ (healthy volunteers); $n=1$ (proband), $n=2$ (homozygous relatives), $n=3$ (unaffected relatives). Normal control ranges are indicated. 
other groups have found that aspirin does not have a significant effect on spreading. ${ }^{33}$

The proband had a more severe bleeding phenotype than the other family members homozygous for the PTGS1 variant which might be attributable to an additional diagnosis of CF and antibiotic use $e^{34}$ but is more likely due to an additional dysfunctional pathway. ${ }^{35,36}$ Indeed, ristocetin-induced platelet aggregation was impaired even though VWB factor antigen (VWF:Ag) and function (VWF:RCo) levels were in the normal range (83.2 IU/dL and 71.7 IU/dL respectively). No coagulation defect was identified which could contribute to bleeding: prothrombin time (9.6 seconds) and activated partial thromboplastin time (28 seconds) were in the normal range and the proband's factor VIII level was $0.98 \mathrm{IU} / \mathrm{mL}$, above the minimum required for normal haemostasis. Furthermore, no variants were found in GP1b or P-selectin in the proband. Interestingly, the proband and homozygous relatives had significant changes in platelet spreading on collagen where $70 \%$ fewer platelets adhered than samples taken from controls, a response which is dependent upon platelet integrin $\alpha_{\mathrm{II}} \beta_{3}$ (which also binds VWF and fibrinogen). Also, of the platelets that did adhere, fewer reached the stage of being fully spread.

As expected, COX-1-deficient platelets in whole blood failed to produce any COX-derived prostanoids, namely $\mathrm{PGE}_{2}, \mathrm{PGD}_{2}, 11$-HETE, 15-HETE and the stable metabolite of $\mathrm{TXA}_{2}\left(\mathrm{TXB}_{2}\right)$ following exposure to platelet agonists. ${ }^{32,37-39}$ Notably, the individuals supplying these platelets had thromboxane metabolite (TX-M) levels within the normal range indicating that urinary TX-M is not a valid or reliable measure of platelet function; contrary to its frequent use for this purpose. This finding supports our recent report that in humans basal TX-M is not derived from platelets but from other sources such as the kidneys,$^{16}$ and provides further rebuttal to challenges of this interpretation. ${ }^{40}$ As urinary TX-M levels are reduced in humans consuming low dose aspirin ${ }^{8}$, the findings also demonstrate that low dose aspirin is not specific for platelets and inhibits COX at other sites. Previous studies have measured the urinary eicosanoid profile in CF patients and reported higher levels of TX-M than in healthy comparators. This is in agreement with our findings in the proband who had higher levels than other homozygous family members. This implies that the elevated production of $\mathrm{TXA}_{2}$ in CF leading to increased TX$M$ cannot be explained by increased platelet activation. ${ }^{41}$ Indeed, COX-2 inhibitors reduced urinary TX-M levels in CF patients consistent with a source other than platelet COX-1.42

Previous cases have been reported variants in PTGS1 which have been associated with autosomal dominant inheritance of enhanced bleeding, some impairment of platelet aggregation and changes in protein levels. There have been no reports of absence of COX-1 protein and/or ablation of associated eicosanoid production as reported here. ${ }^{39,43,52,55,44-51}$ Nance et al. ${ }^{50}$ identified a pedigree with a non-synonymous variant in the signal peptide of PTGS1 (rs3842787; c.50C > T, p.Pro17Leu) that segregated with an aspirin-like platelet function defect. The proband also carried a variant in the F8 causing hemophilia A (rs28935203; c.5096A > T; p.Y1699F). The affected family members with both variants had more severe bleeding than expected from mild hemophilia A alone. In this study, extensive platelet function testing was performed demonstrating impaired platelet aggregation induced by AA, epinephrine and low dose $\mathrm{ADP}$ and reduced platelet $\mathrm{TXB}_{2}$ release. ${ }^{50}$ Two compound heterozygous cases have been reported. The first in a patient with post-procedural bleeds and an aspirin-like defect who carried two high frequency variants (R8L and P17L) which had previously been reported not to have an effect on function. ${ }^{31,52}$ Analysis of the second case identified a rare variant (c.337C>T, p.Arg113Cys; gnomAD frequency $6.134 \times 10^{-5}$ ) in compound heterozygosity with a common variant (c.1003G >A, p.Val481Ile; gnom $A D$ frequency 0.007 ) which was classified as probably pathogenic and accompanied reduced plasma $\mathrm{TXB}_{2}$ levels. ${ }^{51}$ Finally, Bastida et al. reported two cases with variants in PTGS1 (c.35_40delTCCTGC, p.Leu13_Leu14del and c.428A>G, p.Asn143Ser) by sequencing 82 patients with an inherited platelet disorder on their high-throughput sequencing platform to investigate the unknown molecular pathology. They did not, however, perform indepth platelet phenotyping ${ }^{53}$. Consequently, none of these previous reports describe complete loss of platelet PTGS1 function.

In conclusion, we describe the first case of a well characterized family with autosomal recessive inheritance producing an aspirin-like platelet function defect due to a rare variant in PTGS1. This case models the specific loss of platelet COX-1 activity and provides a benchmark of COX1 's role in platelet function and eicosanoid metabolism.

\section{Disclosures}

No conflicts of interest to disclose.

\section{Contributions}

MVC, MAH, SS, MC, MEA, MLE, DCZ, GLM, JS, DG, $M H, V B O, L D, M G M, C L, K W, M S, K D, D P H$ and $K F$ designed and performed experiments; $M V C, M A H, S S, M C$, MEA, MLE, GLM, VBO and LD performed data analysis; $M V C, M A H, S S, M A L$ and TDW wrote the manuscript; VBO, WHO, ET, KF, MAL and TDW supervised the study, and all authors reviewed the manuscript.

\section{Acknowledgments}

The authors would like to thank research nurse Amy Frary at the University of Cambridge for her contributions to the clinical phenotyping of National Institute for Health Research (NIHR) BioResource participants. The authors would also like to thank NIHR BioResource volunteers for their participation, and gratefully acknowledge NIHR BioResource centers, NHS Trusts and staff for their contribution. We thank the National Institute for Health Research and NHS Blood and Transplant. The views expressed are those of the author(s) and not necessarily those of the NHS, the NIHR or the Department of Health and Social Care.

\section{Funding}

The NIHR BioResource was mainly funded by grants from the NIHR in England (NIHR, grant number RG65966). Additional funding was provided by the British Heart Foundation (BHF), Medical Research Council (MRC), National Health Service (NHS) England, the Wellcome Trust and many other fund providers (also see Funding acknowledgment for individual researchers below). This work was supported by the British Heart Foundation (PG/15/47/31591 to TDW for $M V C$, $P G / 17 / 40 / 33028$ to $T D W$ for $M C$, and $R G / 12 / 11 / 29815$ to VBO), Barts \& the London School of Medicine and Dentistry, Queen Mary University of London (to TDW for MAH and 
HEA) and National Institute for Health Research (ACF to SS, Biomedical Research Centres (BRC) 2017-2022: Population and Quantitative Science to DG and ET). This work was supported, in part, by the Division of Intramural Research, National Institute of Environmental Health Sciences, NIH (Z01 ESO25034 to DCZ). VBO is also a Roval Society Wolfson Merit Award Holder and is further supported by the Wellcome Trust
1094143/Z/10/Z) and European Research Council (LipidArrays). This work was supported by National Institutes of Health grant R01 GM115386 (MGM). KD is supported as a HSST trainee by Health Education England. KF is supported by CSL Behring, Baver and SOBI Chairs and Research Council of the University of Leuven (BOF KU Leuven, Belgium, OT/14/098).

\section{References}

1. Allen RD, Zacharski LR, Widirstky ST, Rosenstein R, Zaitlin LM, Burgess DR. Transformation and motility of human platelets. Details of the shape change and release reaction observed by optical and electron microscopy. J Cell Biol. 1979; 83(1):126-142

2. Bearer EL, Prakash JM, Li Z. Actin dynamics in platelets. Int Rev Cytol. 2002; 217:137-182.

3. Brass LF, Wannemacher KM, Ma P, Stalker TJ. Regulating thrombus growth and stability to achieve an optimal response to injury. J Thromb Haemost. 2011;9(1):66-75.

4. Smith WL, DeWitt DL, Garavito RM. Cyclooxygenases: structural, cellular, and molecular biology. Annu Rev Biochem. 2000;69(1):145-182.

5. Smith WL, Garavito RM, DeWitt DL. Prostaglandin endoperoxide $\mathrm{H}$ synthases (cyclooxygenases)-1 and -2. J Biol Chem. 1996;271(52):33157-33160.

6. Smith WL, Urade Y, Jakobsson PJ. Enzymes of the cyclooxygenase pathways of prostanoid biosynthesis. Chemical Rev. 2011:111(10):5821-5865.

7. Ricciotti E, Fitzgerald GA. Prostaglandins and inflammation. Arterioscler Thromb Vasc Biol. 2011;31(5):986-1000.

8. FitzGerald GA, Oates JA, Hawiger J, et al. Endogenous biosynthesis of prostacyclin and thromboxane and platelet function during chronic administration of aspirin in man. J Clin Invest. 1983;71(3):676-688.

9. Patrono C, Coller B, FitzGerald GA, Hirsh J, Roth G. Platelet-active drugs: the relationships among dose, effectiveness, and side effects. Chest. 2004;126(3):234S-264S

10. Warner TD, Nylander S, Whatling C. Antiplatelet therapy: cyclo-oxygenase inhibition and the use of aspirin with particular regard to dual anti-platelet therapy. $\mathrm{Br} \mathrm{J}$ Clin Pharmacol 2011;72(4):619-633.

11. Jaffe EA, Weksler BB. Recovery of endothelial cell prostacyclin production after inhibition by low doses of aspirin. J Clin Invest 1979;63(3):532-535.

12. Westbury SK, Turro E, Greene D, et al. Human phenotype ontology annotation and cluster analysis to unravel genetic defects in 707 cases with unexplained bleeding and platelet disorders. Genome Med. 2015;7(1):36.

13. Ouwehand WH, Gräf S, Tilly T, Swietlik EM, Morrell NW. Whole-genome sequencing of rare disease patients in a national healthcare system. BioRxiv. 2020 Jan 17. [Epub ahead of print]

14. Sanger F, Nicklen S, Coulson AR. DNA sequencing with chain-terminating inhibitors. Proc Natl Acad Sci U S A. 1977; 74(12):5463-5467.

15. Vecchio AJ, Simmons DM, Malkowski MG. Structural basis of fatty acid substrate binding to cyclooxygenase-2. J Biol Chem.
2010·285(29):22152-22163

16. Mitchell JA, Knowles RB, Kirkby NS, et al. Kidney transplantation in a patient lacking cytosolic phospholipase A2proves renal origins of urinary PGI-M and TX-M. Circ Res. 2018;122(4):555-559.

17. Chan M V., Armstrong PCJJ, Papalia F, Kirkby NS, Warner TD. Optical multichannel (optimul) platelet aggregometry in 96well plates as an additional method of platelet reactivity testing. Platelets. 2011;22(7):485-494.

18. Chan M V, Warner TD. Standardised optical multichannel (optimul) platelet aggregometry using high-speed shaking and fixed time point readings. Platelets. 2012; 23(5):404-408.

19. Inoue $O$, Suzuki-Inoue $K$, Dean $W L$, Frampton J, Watson SP. Integrin $\alpha 2 \beta 1$ mediates outside-in regulation of platelet spreading on collagen through activation of Src kinases and PLCY2. I Cell Biol. 2003;160(5):769-780.

20. Kircher M, Witten DM, Jain P, O'roak BJ, Cooper GM, Shendure J. A general framework for estimating the relative pathogenicity of human genetic variants. Nat Genet. 2014;46(3):310-315

21. Lek M, Karczewski KJ, Minikel EV, et al. Analysis of protein-coding genetic variation in 60,706 humans. Nature. 2016;536(7616):285-291.

22. Roberts LJ, Sweetman BJ, Oates JA. Metabolism of thromboxane B2 in man. Identification of twenty urinary metabolites. J Biol Chem. 1981;256(16):8384-8393.

23. McFadyen JD, Peter K. Platelet lipidomics and function: Joining the dots. Blood. 2018; 132(5):465-466.

24. Cathcart M-C, Tamosiuniene R, Chen G, et al. Cyclooxygenase-2-linked attenuation of hypoxia-induced pulmonary hypertension and intravascular thrombosis. J Pharmacol Exp Ther. 2008;326(1):51-58.

25. Derry S, Loke YK. Risk of gastrointestinal haemorrhage with long term use of aspirin: meta-analysis. BMJ. 2000;321(7270):11831187.

26. Lands LC, Stanojevic S. Oral non-steroidal anti-inflammatory drug therapy for lung disease in cystic fibrosis. Cochrane Database Syst Rev. 2016;4(6):CD001505.

27. Vane JR, Botting RM. Mechanism of action of anti-inflammatory drugs. Scand J Rheumatol. 1996;102:9-21.

28. Schubert S, Weyrich AS, Rowley JW. A tour through the transcriptional landscape of platelets. Blood. 2014;124(4):493-502

29. Chan M V., Knowles RBMM, Lundberg $\mathrm{MH}$, et al. P2Y12 receptor blockade synergizes strongly with nitric oxide and prostacyclin to inhibit platelet activation. Br J Clin Pharmacol. 2016;81(4):621-633.

30. Gurbel PA, Bliden KP, DiChiara J, et al. Evaluation of dose-related effects of aspirin on platelet function: results from the Aspirin-Induced Platelet Effect (ASPECT) study. Circulation 2007;115(25):3156-3164.

31. Lee CR, Bottone FG, Krahn JM, et al.
Identification and functional characterization of polymorphisms in human cyclooxygenase-1 (PTGS1). Pharmacogenet Genomics. 2007:17(2):145-160.

32. Loftin CD, Tiano HF, Langenbach $\mathrm{R}$ Phenotypes of the COX-deficient mice indicate physiological and pathophysiological roles for COX-1 and COX-2. Prostaglandins Other Lipid Mediat. 2002; 68-69:177-185

33. Paknikar AK, Eltzner B, Köster S. Direct characterization of cytoskeletal reorganization during blood platelet spreading. Prog Biophys Mol Biol. 2019;144:166-176.

34. Hochman R, Clark J, Rolla A, Thomas S, Kaldany A, D'elia JA. Bleeding in patients with infections: are antibiotics helping or hurting? Arch Intern Med. 1982 142(8):1440-1442

35. Harrison P, Mackie I, Mumford A, et al Guidelines for the laboratory investigation of heritable disorders of platelet function. Br J Haematol. 2011;155(1):30-44.

36. Greene D, Richardson S, Turro E. A fast association test for identifying pathogenic variants involved in rare diseases. Am J Hum Genet. 2017;101(1):104-114

37. Funk CD, Funk LB, Kennedy ME, Pong AS, Fitzgerald GA. Human platelet/erythroleukemia cell prostaglandin $\mathrm{G} / \mathrm{H}$ synthase: cDNA cloning, expression, and gene chromosomal assignment. FASEB J. 1991; 5(9):2304-2312

38. Kirkby NS, Reed DM, Edin ML, et al Inherited human group IVA cytosolic phospholipase A2deficiency abolishes platelet, endothelial, and leucocyte eicosanoid generation. FASEB J. 2015;29(11):4568-4578.

39. Matijevic-Aleksic N, Mcphedran P, Wu KK. Bleeding disorder due to platelet prostaglandin $\mathrm{H}$ synthase-1 (PGHS-1) deficiency. Br J Haematol. 1996;92(1):212-217.

40. Grosser T, Naji A, FitzGerald GA. Urinary prostaglandin metabolites: an incomplete reckoning and a flush to judgment. Circ Res. 2018;122(4):537-539.

41. Strandvik B, Svensson E, Seyberth HW Prostanoid biosynthesis in patients with cystic fibrosis. Prostaglandins Leukot Essent Fat Acids. 1996;55(6):419-425.

42. Ciabattoni G, Davi G, Collura M, et al. In vivo lipid peroxidation and platelet activation in cystic fibrosis. Am J Respir Crit Care Med. 2000;162(4 Pt 1):1195-1201

43. Dubé JN, Drouin J, Aminian M, Plant MH, Laneuville $O$. Characterization of a partial prostaglandin endoperoxide $\mathrm{H}$ synthase- 1 deficiency in a patient with a bleeding disorder. Br J Haematol. 2001;113(4):878-885.

44. Fuse I, Ootsuka T, Hattori A, Mito M Shibata A. Vascular thromboxane formation in hemostasis mechanism: correlation between bleeding time and vascular TXB2 in a patient with congenital platelet cyclooxygenase deficiency. Int J Hematol. 1996;63(4):317-324

45. Horellou MH, Lecompte T, Lecrubier C, et al. Familial and constitutional bleeding disorder due to platelet cyclo-oxygenase defi- 
ciency. Am J Hematol. 1983;14(1):1-9.

46. Lagarde M, Byron PA, Vargaftig BB, Dechavanne M. Impairment of platelet thromboxane A 2 generation and of the platelet release reaction in two patients with congenital deficiency of platelet cyclooxygenase. Br J Haematol. 1978;38(2):251266.

47. Malmsten C, Hamberg M, Svensson J, Samuelsson B. Physiological role of an endoperoxide in human platelets: hemostatic defect due to platelet cyclo-oxygenase deficiency. Proc Natl Acad Sci. 1975;72(4):1446-1450.

48. Nyman D, Eriksson AW, Lehmann W, Blombäck M. Inherited defective platelet aggregation with arachidonate as the main expression of a defective metabolism of arachidonic acid. Thromb Res. 1979;14(45):739-746.

49. Pareti FI, Mannucci PM, D'Angelo A, Smith JB, Sautebin L, Galli G. Congenital deficiency of thromboxane and prostacyclin. Lancet. 1980;315(8174):898-901.

50. Nance D, Campbell RA, Rowley JW, et al. Combined variants in factor VIII and prostaglandin synthase-1 amplify hemorrhage severity across three generations of descendants. J Thromb Haemost 2016; 14(11):2230-2240.

51.Leinøe E, Zetterberg E, Kinalis $\mathrm{S}$, et al. Application of whole-exome sequencing to direct the specific functional testing and diagnosis of rare inherited bleeding disorders in patients from the Öresund Region, Scandinavia. Br J Haematol. 2017; 179(2):308-322.

52. Yagmur E, Weiskirchen R, Schedel A, Bugert P. PTGS1 compound heterozygosity impairs gene expression and platelet aggregation and is associated with severe bleeding complications. Thromb Haemost. 2013;110(5):1083-1085.

53. Bastida JM, Lozano ML, Benito R, et al Introducing high-throughput sequencing into mainstream genetic diagnosis practice in inherited platelet disorders Haematologica. 2018;103(1):148-162. 